\title{
Ginsenoside-Rg5 treatment inhibits apoptosis of chondrocytes and degradation of cartilage matrix in a rat model of osteoarthritis
}

\author{
PING ZHANG \\ The Disease Prevention Center of Anyang Hospital of Traditional Chinese \\ Medicine of Henan Province, Nanyang, Henan 455000, P.R. China
}

Received June 19, 2016; Accepted November 7, 2016

DOI: 10.3892/or.2017.5392

\begin{abstract}
This study investigated the effect of ginsenoside-Rg5 on the degradation of articular cartilage in osteoarthritis rat model and on induction of chondrocyte apoptosis. Osteoarthritis rat model was prepared by ligament transection and medial meniscus resection. The rats were then treated with different doses $(1,2,5,10$ and $15 \mu \mathrm{M})$ of ginsenoside- $\operatorname{Rg} 5$ for $48 \mathrm{~h}$. The results from histopathological analysis revealed a significant $(\mathrm{P}=0.005)$ prevention of cartilage degradation in OA rat model by ginsenoside- $\operatorname{Rg} 5$ treatment at $15 \mu \mathrm{M}$. Ginsenoside-Rg5 treatment prevented the disintegration of synovial membrane to a significant $(\mathrm{P}=0.005)$ extent. The proportion of apoptotic cells in the knee joints was reduced to $7 \%$ by ginsenoside-Rg 5 treatment after one month compared to the control. Treatment of the rats with ginsenoside-Rg 5 caused increase in the levels of proteoglycan, collagen and type II collagen by 5-, 3- and 4-fold compared to the control group. Immunohistochemistry revealed that the level of MMP-13 was reduced to $45 \%$ and that of TIMP-1 was increased by $67 \%$ on treatment with ginsenoside-Rg5. The levels of interleukin-1 $\beta$, tumor necrosis factor- $\alpha$, nitric oxide and inducible nitric oxide synthetase were reduced by $67,54,32$ ad $49 \%$, respectively after one month of treatment with $15 \mathrm{mg} / \mathrm{kg}$ dose of ginsenoside- $\mathrm{Rg} 5$. The expression was increased to 67 and 52\% for BMP-2 and TGF- $\beta 1$, respectively on treatment with ginsenoside-Rg5. Thus ginsenoside- $\operatorname{Rg} 5$ prevents cartilage degradation in the OA rats and inhibits cartilage apoptosis, therefore it can be used for osteoarthritis treatment.
\end{abstract}

\section{Introduction}

Osteoarthritis (OA) is one of the commonly observed diseases of joints caused by the disintegration of cartilage matrix and

Correspondence to: Dr Ping Zhang, The Disease Prevention Center of Anyang Hospital of Traditional Chinese Medicine of Henan Province, 150 Hongqi Road, Nanyang, Henan 455000, P.R. China E-mail: drzhangping@hotmail.com

Key words: osteoarthritis, apoptosis, cartilage, necrosis, ligament death of chondrocytes (1). There is always an equilibrium maintained by extracellular cartilage matrix between the formation and loss of chondrocytes $(2,3)$. Loss of chondrocytes is a limiting factor for the formation of extracellular cartilage matrix which subsequently leads to the development of osteoarthritis (4). Many factors such as proteoglycan and collagen (type II) are involved in the regulation of normal functioning of chondrocytes (5). Studies have revealed that chondrocyte loss and extracellular matrix degradation involve several factors including, generation of peroxide radicals, TNF- $\alpha$ and interleukins $(6,7)$. All these factors initiate inflammatory reactions leading to chondrocyte apoptosis (8). Expression of interleukin- $1 \beta$ is responsible for the production of inhibitors of metalloproteinase (TIMPs) leading to the inhibition of matrix metalloproteinase (MMP) generation and subsequent chondrocyte death and OA (9-11).

Traditional Chinese medicine (TCM) involves the use of various herbs as well as dietary ingredients for several disorders depending upon the type of the syndrome (12). Numerous studies have been performed to demonstrate the chemotherapeutic potential of TCM against various types of cancers (13). Ginseng has a long traditional medicinal importance for the treatment of cancers, stress and diabetes (14). Ginsenoside-Rg5 obtained by the processing of ginseng has shown promising anticancer, anti-inflammatory and anti-aging properties (15-17). There are several reports which demonstrate the activity of ginsenoside-Rg5 against cancer $(18,19)$, dermatis (20), inflammation (21) and neurotrophic disorders (22). This study was performed to investigate the effects of ginsenoside-Rg5 on destruction of cartilage through onset of cartilage matrix damage and death of chondrocytes in OA rat model. This study revealed that ginsenoside-Rg5 significantly prevents destruction of articular cartilage through inhibition of chondrocyte apoptosis and matrix damage in OA rats.

\section{Materials and methods}

Animals. Male adult Wistar rats aged 8-10 weeks (270-300 g) were purchased from the Laboratory Animal Center of Sun Yat-Sen University and were housed in the animal care facility center of our institute under pathogen-free conditions. The experimental procedures on animals were performed according to the guidelines of the National Institutes of Health criteria for the care and use of laboratory animals. This study 
was approved by the Laboratory Animal Care Committee of Sun Yat-sen University (Guangzhou, China).

Preparation of osteoarthritis rat model. The animals were subjected to anesthetization using halothane, knee joint was exposed to dislocate the patella and subsequently microscissors were used for transection of ligament and resection of the medial meniscus. The rats were randomly assigned to seven groups of 5 each. The five treatment groups were given $1,2,5,10$ or $15 \mathrm{mg} / \mathrm{g}$ doses of ginsenoside- $\operatorname{Rg} 5$ intragastrically daily for one month. The rats in the normal and untreated groups received equal volume of normal saline at the same time.

Histological analysis. On day 31 animals were sacrificed to extract the tibia and femur bones from each of the animals. The dissected bones were subjected to fixing in paraformaldehyde followed by decalcification and paraffin embedment. The bones were then cut into thin sections, deparaffined and subjected to hematoxylin and eosin staining for microscopic examination. The cartilage damage was monitored on the Mankin scale ranging from 0 to 12 . The quantification of synovial lining damage was performed using Image-Pro Plus 6.0 system (IPP) image analysis system (Media Cybernetics, Rockville, MD, USA).

Terminal deoxynucleotidyl transferase-mediated deoxyuridine triphosphate nick-end labeling (TUNEL) staining. Antigen retrieval was conducted according to the manufacturer's instructions (Roche) and after dewaxing. The cartilage sections were washed twice with PBST and then permeabilized using $0.1 \%$ Triton $\mathrm{X}-100$. Incubation of the sections was performed using fluorescein-labeled dUTP and terminal deoxynucleotidyl transferase (TdT) mixture. Antifluorescein antibody in combination with alkaline phosphatase was used for probing purposes. The Olympus OX31 microscope (Olympus, Tokyo, Japan) was used for the analysis of the apoptosis of the chondrocytes. Quantification of the apoptotic chondrocytes was performed using Image-Pro Plus 6.0 system (IPP).

Immunoblotting analysis. The paraffin-embedded sections were cut into thin sections followed by de-paraffinization in boiling xylene. The sections were treated by hydrogen peroxide and then incubated with blocking serum (Vectastain ${ }^{\circledR}$ ABC kit, Vector Laboratories, Burlingame, CA, USA) for $45 \mathrm{~min}$. The sections were incubated with mouse monoclonal antibodies against type II collagen, MMP-13 and TIMP-1 (dilution 1/30; Beijing Biosynthesis Biotechnology Co., Ltd., Beijing, China) overnight at $4^{\circ} \mathrm{C}$. The sections were washed with PBS an subsequently incubated with horse secondary antibody (Zhonshan Golden Bridge Biotechnology, Beijing, China) for $1 \mathrm{~h}$. The 3,3'-diaminobenzidine (DAB; Sigma, St. Louis, MO, USA) stained sections were analyzed using Image-Pro Plus 6.0 system (IPP).

Flow cytometry for analysis of apoptosis induction. Chondrocytes treated with ginsenoside-Rg5 were collected, rinsed in ice-cold PBS. Chondrocytes were then suspended at a density of $2 \times 10^{6}$ per ml into the binding buffer and subjected to incubation. Then $100 \mathrm{ml}$ samples were put into the fulcrum tubes and treated with Annexin V-FITC (5 $\mu \mathrm{l})$ and propidium iodide $(10 \mu \mathrm{l})$ for $30 \mathrm{~min}$ under dark atmosphere. Following incubation, chondrocytes were treated with binding buffer and then analyzed using flow cytometry.

Western blot assay. After incubation of the chondrocytes with ginsenoside-Rg5 for 48 h, $300 \mu 1$ lysis buffer (Wuhan Boster Biological Technology, Ltd., Wuhan, China) was added. Then cell lysate was treated with $3 \mu$ l protease inhibitor and the mortar for $45 \mathrm{~min}$ on ice followed by centrifugation for $30 \mathrm{~min}$ at $12,000 \mathrm{x}$. The supernatant was collected for the determination of concentration of proteins. To this mixture was added $4 \mathrm{X}$ sample buffer solution, followed by boiling for $45 \mathrm{~min}$. The centrifuged mixture was subjected to isolation on SDS-PAGE and the protein transfer to polyvinylidene difluoride membranes. The membranes were blocked using $5 \%$ skimmed milk and subsequently incubated with mouse anti-MMP-13 monoclonal antibody (1:100; sc-189; Santa Cruz Biotechnology, Inc., Santa Cruz, CA, USA) and rabbit anti-TIMP-1 polyclonal antibody (1:100; sc-189; Santa Cruz Biotechnology, Inc.). After overnight incubation, the membranes were washed twice with PBS and then incubated for $1 \mathrm{~h}$ with horseradish peroxide-coupled sheep anti-mouse secondary antibody (Beijing Kangwei Technology Group Co., Ltd., Beijing, China). The blots were treated with chemiluminescent reagent (GS009; Beyotime Institute of Biotechnology, Shanghai, China) and then images were captured.

Quantitative polymerase chain reaction ( $q P C R)$. The chondrocytes were treated with ginsenoside-Rg5 for 48 h. Following incubation, total RNA from the chondrocytes was isolated using RNA extraction kit (Invitrogen Life Technologies, Carlsbad, CA, USA). After extraction and subsequent purification, the RNA concentration was measured using a spectrophotometer. The $2-\mu \mathrm{g}$ RNA samples were then subjected to reverse transcription using reverse transcription reagent (Takara Biotechnology Co.,Ltd.,Dalian, China).Quantification and analysis of the transcripts was performed using QuantiTect SYBR Green PCR kit (Qiagen, Tokyo, Japan) and Applied Biosystems ${ }^{\circledR} 7500$ Fast Real-Time PCR system (Applied Biosystems Inc.), respectively. PCR was performed using the following reaction conditions: pre-denaturation was performed at $95^{\circ} \mathrm{C}$ for $30 \mathrm{sec}$; at $95^{\circ} \mathrm{C}$ denaturation was carried out for $3 \mathrm{sec}$; and at $60^{\circ} \mathrm{C}$ annealing was done for $30 \mathrm{sec}$. The data were obtained directly from the real-time fluorescent quantitative PCR instrument (Bio-Rad Laboratories, Inc., Hercules, CA, USA). PCR primers used were: TGAGGA TACAGGCAAGACTCT (forward), CAATACGGTTACTCC AGATGC (reverse) for MMP-13 and CTTCTGGCATCCTGT TGTTG (forward), AGAAGGCCGTCTGTGGGT (reverse) for TIMP-1.

Statistical analysis. The data presented were analyzed using SPSS software, version 12.0 (SPSS, Inc., Chicago, IL, USA). Analysis of the data was performed using one-way analysis of variance and subsequent Tukey post hoc comparisons. Statistically significant differences were considered at a two-tailed $\mathrm{P}<0.05$. 
A

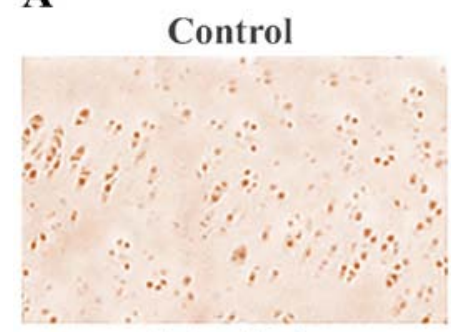

$5(\mathrm{mg} / \mathrm{kg})$
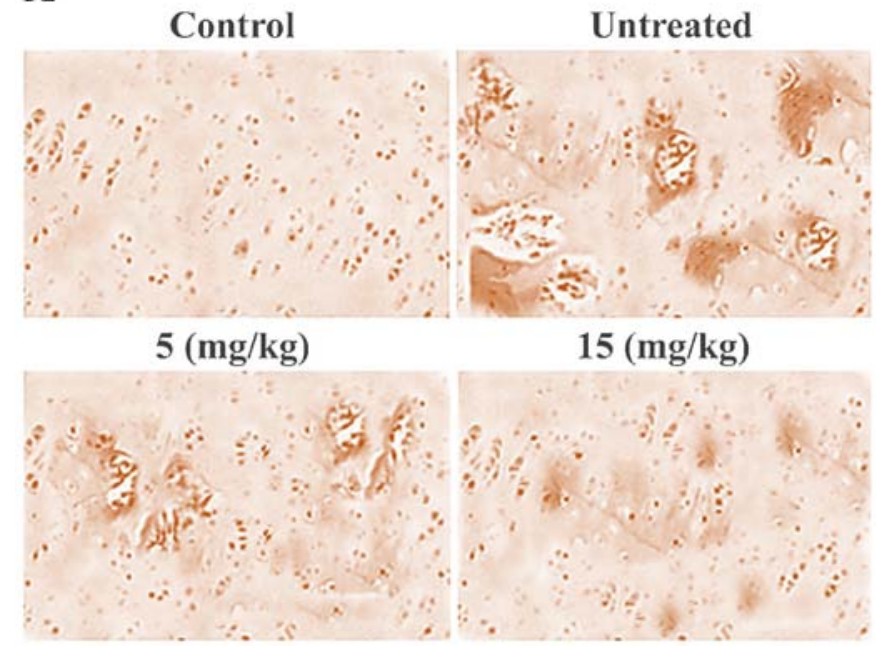

$15(\mathrm{mg} / \mathrm{kg})$
B

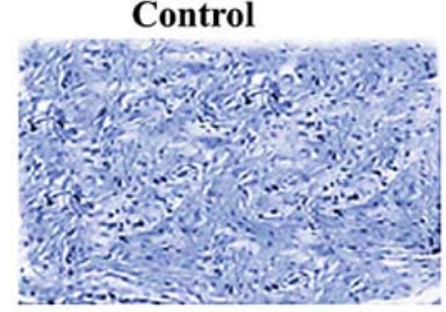

$5(\mathrm{mg} / \mathrm{kg})$

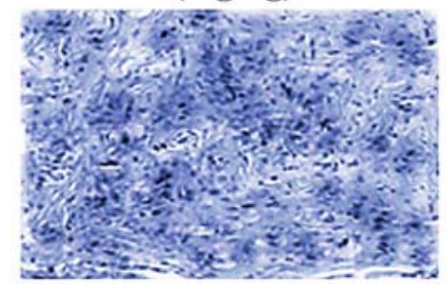

Untreated

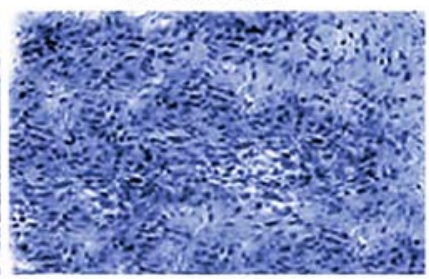

$15(\mathrm{mg} / \mathrm{kg})$

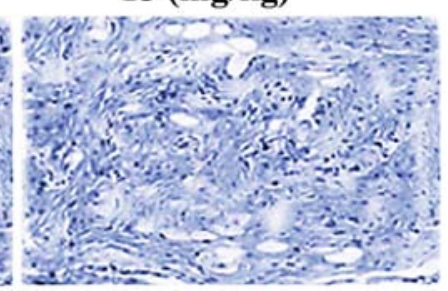

Figure 1. Ginsenoside-Rg5 treatment prevents degradation of (A) knee cartilage and (B) synovium in OA rat model. The rats were sacrificed after one month of treatment with ginsenoside-Rg5. The degradation of cartilage and synovium was examined. The photomicrographs were captured at a magnification of $\mathrm{x} 200$.

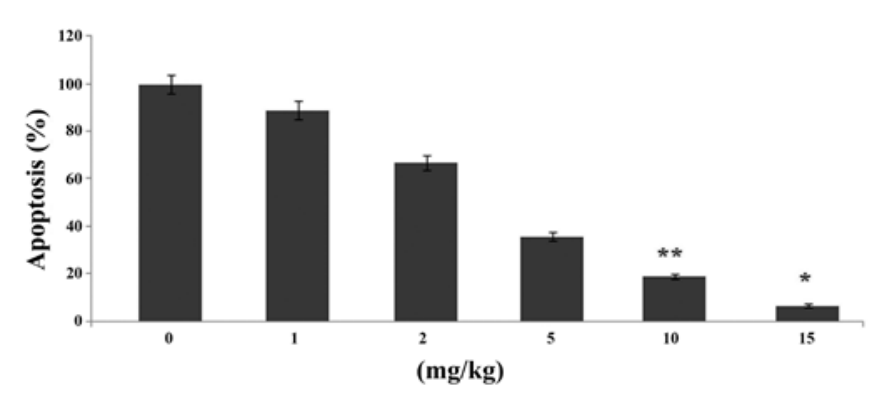

Figure 2. Ginsenoside-Rg5 inhibits induction of apoptosis in the knee chondrocytes in the OA rats. The rats were treated with various doses of ginsenoside-Rg5 for one month and then sacrificed to determine the apoptosis induction in chondrocytes. The presented data are the mean \pm standard deviation of the three experiments performed independently. ${ }^{*} \mathrm{P}<0.05$ and ${ }^{* *} \mathrm{P}<0.001$ verses control group.

\section{Results}

Ginsenoside-Rg5 treatment prevents damage to articular knee cartilage in OA-rat model. Histopathological analysis of the OA-rat cartilage revealed marked changes such as presence of wide spaces, dissociated cells and rough surface. However, ginsenoside- $\operatorname{Rg} 5$ treatment prevented damage to cartilage in the OA-rats following one month of treatment (Fig. 1A). Among the various doses used to treat the OA-rats, the effect was significant $(\mathrm{P}=0.005)$ at $15 \mathrm{mg} / \mathrm{kg}$ concentration of ginsenoside-Rg5. In comparison to the untreated group of OA-rats, the Mankin score in the ginsenoside-Rg5 treated group was significantly lower. Synovial examination showed irregular surface and higher population of the inflammatory cells in the OA-rats. However, ginsenoside-Rg5 treatment prevented the disintegration of synovial membrane to a significant $(\mathrm{P}=0.005)$ extent compared to the untreated group (Fig. 1B).

Effects of ginsenoside-Rg5 on knee chondrocyte apoptosis in the OA-rat model. Ginsenoside-Rg5 treatment caused a significant $(\mathrm{P}<0.05)$ reduction in the proportion of apoptotic cells in the cartilage compared to the control group (Fig. 2). Ginsenoside-Rg5 treatment for one month caused reduction in the proportion of apoptotic cells to $7 \%$ in the knee joints compared to the control.

Effects of ginsenoside-Rg5 on knee cartilage matrix in the OA-rat model. Treatment of the rats with ginsenoside-Rg5 for one month caused a significant prevention of the degradation of cartilage matrix compared to those in the control group. The levels of proteins including, proteoglycan, collagen and type II collagen were increased by 5-, 3- and 4-fold compared to the control group (Fig. 3).

Ginsenoside-Rg5 treatment alters the expression levels of MMP-13 and TIMP-1 in the OA-rat model. Immunohistochemistry revealed a significant alteration in the expression levels of MMP-13 and TIMP-1 in the rats on treatment with ginsenoside-Rg5 compared to the control group after one month. The level of MMP-13 was reduced to $45 \%$ compared to the control and that of TIMP-1 was increased by $67 \%$ in the rats treated with ginsenoside- $\operatorname{Rg} 5$ for one month (Fig. 4).

Ginsenoside-Rg5 treatment alters the expression of interleukin-1 $\beta$, tumor necrosis factor- $\alpha$, nitric oxide, inducible nitric oxide synthetase, BMP and TGF- $\beta$ in the serum of $O A$-rat model. Treatment of the OA-rats with ginsenoside-Rg5 for one month caused a significant reduction in the expression levels of interleukin-1 $\beta$, tumor necrosis factor- $\alpha$, nitric oxide and inducible nitric oxide synthetase. The levels of interleukin-1 $\beta$, tumor necrosis factor- $\alpha$, nitric oxide and inducible nitric oxide synthetase were reduced by $67,54,32$ ad $49 \%$, respectively, after one month of treatment with $5 \mathrm{mg} /$ $\mathrm{kg}$ dose of ginsenoside-Rg5. Ginsenoside-Rg5 treatment for one month led to a significant enhancement in the expression levels of BMP-2 and TGF- $\beta 1$ in the OA-rats. The levels were increased to 67 and 52\% for BMP-2 and TGF- $\beta 1$, respectively after one month (Fig. 5). 


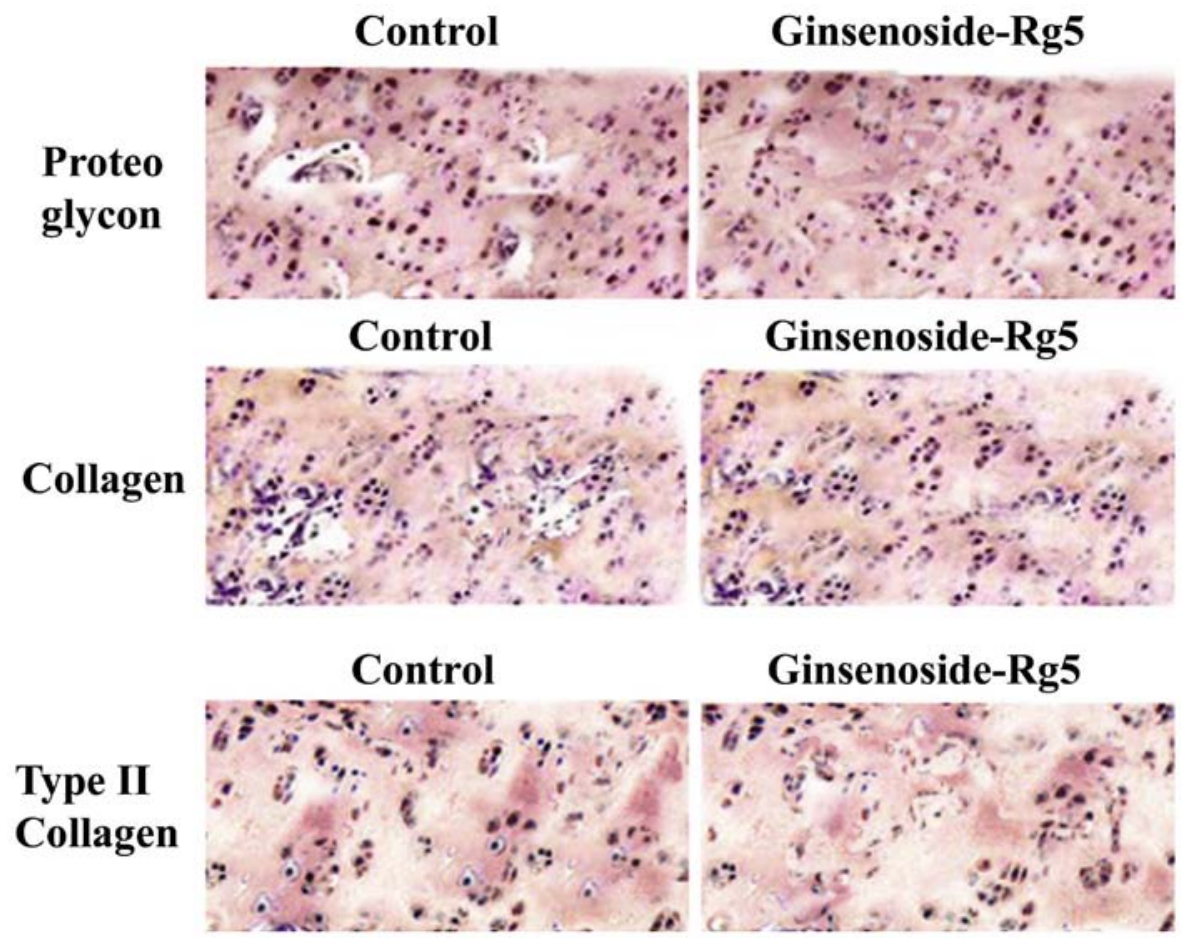

Figure 3. Ginsenoside-Rg5 prevents extracellular cartilage matrix degradation in the OA rats. In the rats treated with various doses of ginsenoside-Rg5 for one month the levels of proteoglycan, collagen and type II collagen were analyzed. The photomicrographs were captured at a magnification of x200.
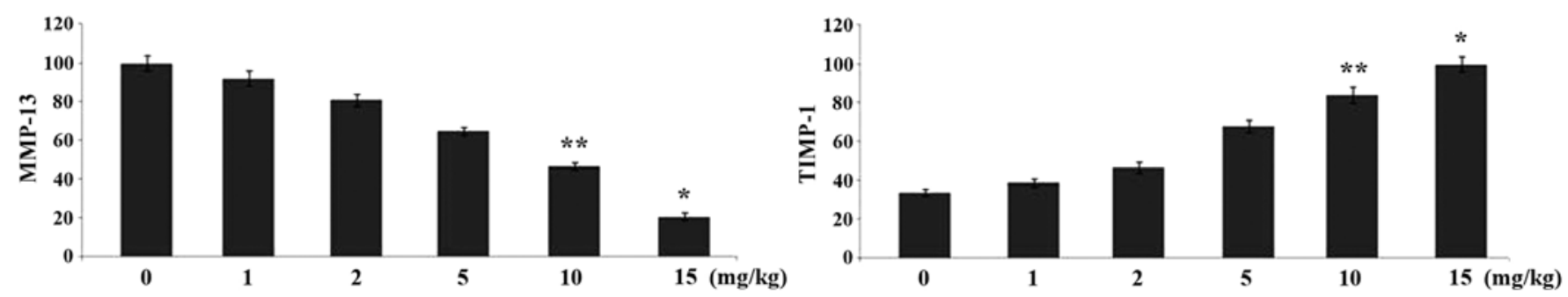

Figure 4. Ginsenoside-Rg5 alters the expression level of matrix metalloprotein-13 and its endogenous inhibitor-1 in the OA rats. The rats were treated with ginsenoside-Rg5 for one month and then the expression of matrix metalloprotein- 13 and its endogenous inhibitor- 1 were examined. Images were captured at a magnification of $\times 200$.

Ginsenoside-Rg5 treatment inhibits chondrocyte apoptosis. Incubation of the chondrocytes with interleukin- $1 \beta$ caused a marked increase in the proportion of apoptotic cells. However, treatment of the chondrocytes with 1,2,5, 10 and $15 \mu \mathrm{M}$ doses of ginsenoside- $\operatorname{Rg} 5$ for $48 \mathrm{~h}$ prevented the interleukin- $1 \beta$ induced apoptosis of chondrocytes in a dose-dependent manner (Fig. 6). Apoptosis of chondrocytes was reduced to $56,42,29,11$ and $2 \%$ on treatment with $1,2,5,10$ and $15 \mu \mathrm{M}$, respectively doses of ginsenoside-Rg5 after $48 \mathrm{~h}$.

Ginsenoside-Rg5 treatment of chondrocytes alters the expression of MMP-13 and TIMP-1. Treatment of chondrocytes with ginsenoside-Rg5 caused a concentration-dependent decrease in the interleukin-1 $\beta$ induced expression of MMP-13. The expression of MMP-13 was reduced to 82, 76, 58, 34 and $12 \%$ on treatment of chondrocytes with $1,2,5,10$ and $15 \mu \mathrm{M}$, respectively, doses of ginsenoside-Rg5 for $48 \mathrm{~h}$. Treatment of the chondrocytes with ginsenoside- $\operatorname{Rg} 5$ caused a significant increase in the interleukin-1 $\beta$ inhibited expression of TIMP-1
(Fig. 7). Chondrocytes were treated with 1, 2, 5, 10 and $15 \mu \mathrm{M}$ doses of ginsenoside-Rg5 and the expression of TIMP-1 was found to be 2, 3, 3.7, 4.5 and 6-fold, respectively compared to the interleukin-1 $\beta$ treated chondrocytes (Fig. 7).

\section{Discussion}

This study was performed to investigate the effects of ginsenoside-Rg5 on destruction of cartilage through onset of cartilage matrix damage and death of chondrocytes in OA rat model. The results from this study demonstrated that ginsenoside- $\operatorname{Rg} 5$ prevents degradation of cartilage and inhibits inflammation of synovium in the OA rats. OA is characterized by the dissociation and death of chondrocytes, damage to extracellular cartilage matrix and production of free radical leading to inflammatory reactions.

During OA a marked loss of chondrocytes is observed in the articular cartilage which is evident by the formation of wide spaces and rough cartilage surface (23). The current 
A
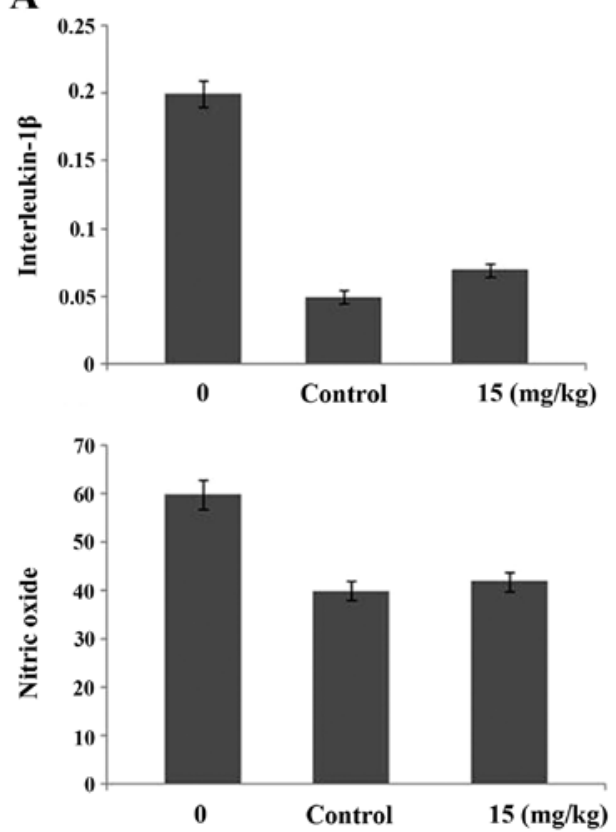

B

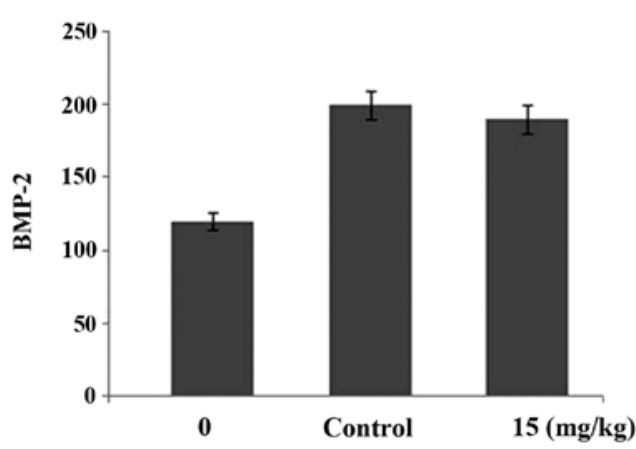

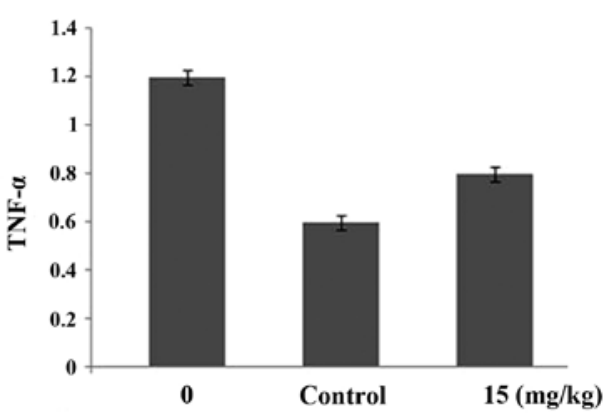

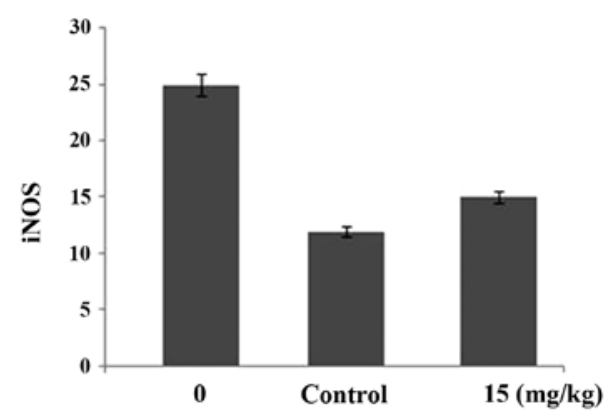

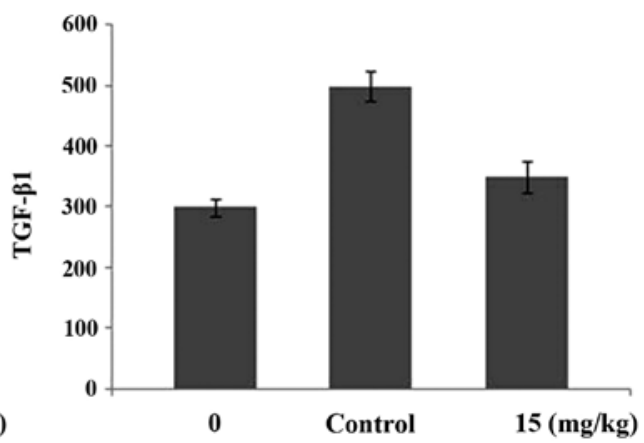

Figure 5. Ginsenoside-Rg5 changes the expression of interleukin-1 $\beta$, tumor necrosis factor- $\alpha$, nitric oxide, inducible nitric oxide synthetase, bone morphogenetic protein and transforming growth factor- $\beta 1$ in the OA rat serum. Treatment of the rats with ginsenoside-Rg5 for one month was followed by analysis of the expression of various factors.

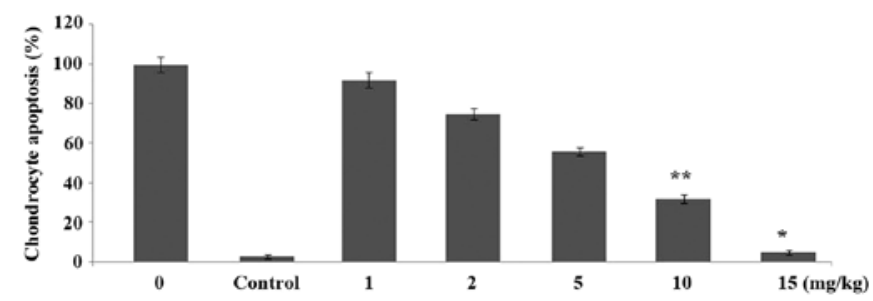

Figure 6. Ginsenoside-Rg5 treatment for $48 \mathrm{~h}$ inhibits induction of chondrocyte apoptosis. The cells were incubated with various concentrations of ginsenoside- $\operatorname{Rg} 5$ and then subjected to flow cytometry.

study revealed a significant increase in the chondrocyte apoptosis and degradation of extracellular matrix in the OA rats. However, ginsenoside-Rg5 treatment led to inhibition of chondrocyte apoptosis and prevention of extracellular matrix damage in OA rats after one month. Apoptosis was also induced in the chondrocytes by incubation with interleukin- $1 \beta$ and the cells were then treated with ginsenoside-Rg5. The results revealed that ginsenoside- $\operatorname{Rg} 5$ treatment significantly

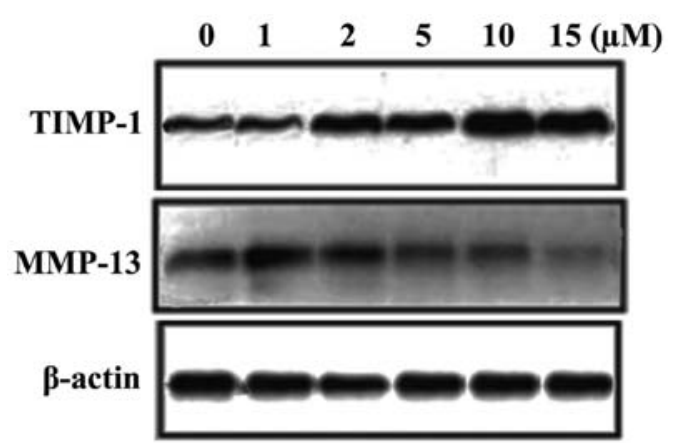

Figure 7. Ginsenoside-Rg5 treatment for $48 \mathrm{~h}$ changes the expression level of matrix metalloprotein-13 and its endogenous inhibitor- 1 in the interleukin-1 $\beta$ treated chondrocytes. The cells were incubated for $48 \mathrm{~h}$ with ginsenoside-Rg5 and then subjected to western blot analysis.

inhibited the interleukin-1 $\beta$ induced apoptosis in the chondrocytes. Degradation of cartilage is caused by the inhibition of proteoglycan which is the major component of extracellular matrix and provides it strength $(24,25)$. Another factor of extra- 
cellular matrix providing strength to the articular is the type II collagen (26). The degradation of type II collagen is caused by MMP-13, however, its activity is suppressed by TIMP-1 $(27,28)$. Our current study revealed that ginsenoside-Rg5 treatment for one month significantly increased the expression of TIMP-1. The increased expression of TIMP-1 was evident by a marked reduction in the MMP-13 expression in OA-rats. Treatment of the chondrocytes with interleukin- $1 \beta$ caused a significant reduction in the expression of TIMP-1. However, treatment of the chondrocytes with ginsenoside- $\operatorname{Rg} 5$ led to a marked increase in the expression of TIMP-1 in following incubation with interleukin-1 $\beta$. Initial stage of $\mathrm{OA}$ is characterized by the inflammation and higher level of proinflammatory cytokines such as interleukin-1 $\beta$ and the tumor necrosis factor (29). In addition, the level of nitric oxide radical and inducible nitric oxide synthetase is also higher which induced chondrocyte death (30). Our results revealed that ginsenoside- $\operatorname{Rg} 5$ treatment caused a significant reduction in the expression of interleukin-1 $\beta$, tumor necrosis factor, nitric oxide radical and inducible nitric oxide synthetase in the OA-rats after one month.

Thus, this study revealed that ginsenoside-Rg5 significantly prevents destruction of articular cartilage through inhibition of chondrocyte apoptosis and matrix damage in OA rats. Therefore, ginsenoside- $\operatorname{Rg} 5$ can be used for the treatment of osteoarthritis.

\section{References}

1. Loeser RF: Aging and osteoarthritis: The role of chondrocyte senescence and aging changes in the cartilage matrix. Osteoarthritis Cartilage 17: 971-979, 2009.

2. Goldring MB: The role of the chondrocyte in osteoarthritis. Arthritis Rheum 43: 1916-1926, 2000.

3. Roughley PJ: Articular cartilage and changes in arthritis: Noncollagenous proteins and proteoglycans in the extracellular matrix of cartilage. Arthritis Res 3: 342-347, 2001.

4. Aigner T and Kim HA: Apoptosis and cellular vitality: Issues in osteoarthritic cartilage degeneration. Arthritis Rheum 46 1986-1996, 2002.

5. Eyre D: Collagen of articular cartilage. Arthritis Res 4: 30-35, 2002.

6. Aizawa T, Kon T, Einhorn TA and Gerstenfeld LC: Induction of apoptosis in chondrocytes by tumor necrosis factor-alpha. J Orthop Res 19: 785-796, 2001.

7. Fernandes JC, Martel-Pelletier J and Pelletier JP: The role of cytokines in osteoarthritis pathophysiology. Biorheology 39: 237-246, 2002.

8. Kapoor M, Martel-Pelletier J, Lajeunesse D, Pelletier JP and Fahmi H: Role of proinflammatory cytokines in the pathophysiology of osteoarthritis. Nat Rev Rheumatol 7: 33-42, 2011.

9. Attur M, Al-Mussawir HE, Patel J, Kitay A, Dave M, Palmer G, Pillinger $\mathrm{MH}$ and Abramson SB: Prostaglandin E2 exerts catabolic effects in osteoarthritis cartilage: Evidence for signaling via the EP4 receptor. J Immunol 181: 5082-5088, 2008.

10. Abramson SB: Osteoarthritis and nitric oxide. Osteoarthritis Cartilage 16 (Suppl 2): S15-S20, 2008.

11. Burger D, Rezzonico R, Li JM, Modoux C, Pierce RA, Welgus HG and Dayer JM: Imbalance between interstitial collagenase and tissue inhibitor of metalloproteinases 1 in synoviocytes and fibroblasts upon direct contact with stimulated T lymphocytes: Involvement of membrane-associated cytokines. Arthritis Rheum 41: 1748-1759, 1998.
12. Yang G, Li X, Li X, Wang L, Li J, Song X, Chen J, Guo Y, Sun X, Wang S, et al: Traditional Chinese medicine in cancer care: A review of case series published in the chinese literature. Evid Based Complement Alternat Med 2012: 751046, 2012.

13. Liu J, Li X, Liu J, Ma L, Li X and Fønneb $\varnothing$ V: Traditional Chinese medicine in cancer care: A review of case reports published in Chinese literature. Forsch Komplement Med 18: 257-263, 2011.

14. Kwon SW, Han SB, Park IH, Kim JM, Park MK and Park JH: Liquid chromatographic determination of less polar ginsenosides in processed ginseng. J Chromatogr A 921: 335-339, 2001.

15. Kim SN, Ha YW, Shin H, Son SH, Wu SJ and Kim YS: Simultaneous quantification of 14 ginsenosides in Panax ginseng C.A. Meyer (Korean red ginseng) by HPLC-ELSD and its application to quality control. J Pharm Biomed Anal 45: 164-170, 2007.

16. Yun TK, Lee YS, Lee YH, Kim SI and Yun HY: Anticarcinogenic effect of Panax ginseng C.A. Meyer and identification of active compounds. J Korean Med Sci 16 (Suppl): S6-S18, 2001.

17. Kang KS, Kim HY, Baek SH, Yoo HH, Park JH and Yokozawa T: Study on the hydroxyl radical scavenging activity changes of ginseng and ginsenoside-Rb2 by heat processing. Biol Pharm Bull 30: 724-728, 2007.

18. Nag SA, Qin JJ, Wang W, Wang MH, Wang H and Zhang R: Ginsenosides as anticancer agents: In vitro and in vivo activities, structure-activity relationships, and molecular mechanisms of action. Front Pharmacol 3: 25, 2012.

19. Lee KY, Lee YH, Kim SI, Park JH and Lee SK: Ginsenoside-Rg5 suppresses cyclin E-dependent protein kinase activity via up-regulating $\mathrm{p} 21^{\mathrm{Cip} / \mathrm{WAF} 1}$ and down-regulating cyclin $\mathrm{E}$ in SK-HEP-1 cells. Anticancer Res 17A: 1067-1072, 1997.

20. Shin YW, Bae EA and Kim DH: Inhibitory effect of ginsenoside $\mathrm{Rg} 5$ and its metabolite ginsenoside $\mathrm{Rh} 3$ in an oxazolone-induced mouse chronic dermatitis model. Arch Pharm Res 29: 685-690, 2006.

21. Kim TW, Joh EH, Kim B and Kim DH: Ginsenoside Rg5 ameliorates lung inflammation in mice by inhibiting the binding of LPS to toll-like receptor-4 on macrophages. Int Immunopharmacol 12: 110-116, 2012.

22. Kim EJ, Jung IH, Van Le TK, Jeong JJ, Kim NJ and Kim DH: Ginsenosides Rg5 and Rh3 protect scopolamine-induced memory deficits in mice. J Ethnopharmacol 146: 294-299, 2013.

23. Kühn K, D'Lima DD, Hashimoto S and Lotz M: Cell death in cartilage. Osteoarthritis Cartilage 12: 1-16, 2004.

24. Jubb RW and Fell HB: The breakdown of collagen by chondrocytes. J Pathol 130: 159-167, 1980.

25. Rosenberg AE: Bones, joints, and soft tissue tumors. In: Robbins Pathologic Basis of Disease. 6th edition. Cotran RS, Kumar C and Collins T (eds). WB Saunders, Philadelphia, PA, p1253, 1999.

26. Naito K, Watari T, Muta T, Furuhata A, Iwase H, Igarashi M, Kurosawa H, Nagaoka I and Kaneko K: Low-intensity pulsed ultrasound (LIPUS) increases the articular cartilage type II collagen in a rat osteoarthritis model. J Orthop Res 28: 361-369, 2010.

27. Goldring MB, Otero M, Plumb DA, Dragomir C, Favero M, El Hachem K, Hashimoto K, Roach HI, Olivotto E, Borzì RM, et al: Roles of inflammatory and anabolic cytokines in cartilage metabolism: Signals and multiple effectors converge upon MMP-13 regulation in osteoarthritis. Eur Cell Mater 21: 202-220, 2011.

28. Wetzel M, Li L, Harms KM, Roitbak T, Ventura PB, Rosenberg GA, Khokha R and Cunningham LA: Tissue inhibitor of metalloproteinases-3 facilitates Fas-mediated neuronal cell death following mild ischemia. Cell Death Differ 15: 143-151, 2008.

29. Hashimoto S, Nishiyama T, Hayashi S, Fujishiro T, Takebe K, Kanzaki N, Kuroda R and Kurosaka M: Role of p53 in human chondrocyte apoptosis in response to shear strain. Arthritis Rheum 60: 2340-2349, 2009.

30. Del Carlo M Jr and Loeser RF: Nitric oxide-mediated chondrocyte cell death requires the generation of additional reactive oxygen species. Arthritis Rheum 46: 394-403, 2002. 